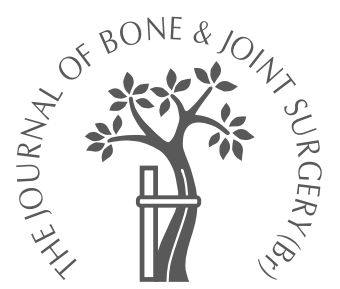

T. Maruta,

S. Minami,

H. Kitahara,

K. Isobe, Y. Otsuka,

Y. Nakata,

H. Moriya

From the Chiba

University, Japan

T. Maruta, MD

Orthopaedic Surgeon

S. Minami, MD, Associate

Professor

K. Isobe, MD, Professor

H. Moriya, MD, Professor

and Chairman

H. Kitahara, MD, Director and Orthopaedic Surgeon

Chiba Rehabilitation Centre,

1-45-2 Hondacho, Midori-Ku,

Chiba 266-0005, Japan.

Y. Otsuka, MD, Director and Orthopaedic Surgeon

Y. Nakata, MD, Consultant Orthopaedic Surgeon

Department of Orthopaedic

Surgery, National Chiba-

Higashi Hospital, 673

Nitonacho, Chuo-Ku, Chiba

260-0801, Japan.

Correspondence should be sent to $\mathrm{Dr} \mathrm{T}$. Maruta.

(C)2004 British Editorial Society of Bone and

Joint Surgery

doi:10.1302/0301-620X.86B2

$14178 \$ 2.00$

$J$ Bone Joint Surg $[\mathrm{Br}]$

2004;86-B:220-4.

Received 17 January 2003;

Accepted after revision

14 July 2003

\section{Rotation of the spinal cord in idiopathic scoliosis}

We undertook a radiographic analysis with pre-operative computed tomographic myelography in $\mathbf{7 8}$ patients with idiopathic scoliosis in order to analyse rotation of the spinal cord and to investigate its clinical significance. The angle of rotation of the cord had a statistically significant relationship to both that of the apical vertebra and the size of the primary curve. The relationship between the rotation of the cord and that of the apical vertebra was divided into three types. The cord rotated in the same direction as the apical vertebra in $\mathbf{5 5}$ patients and rotated in the opposite direction in the remaining 23 patients. In the first group, the angle of rotation of the cord was more than that of the vertebra in six patients, but less than it in $\mathbf{4 9}$ patients. These results suggest that the neuraxis in idiopathic scoliosis may be under tension in the axial dimension.

Idiopathic scoliosis is a multiplanar deformity of the trunk accompanied by lateral deviation, apical lordosis and axial rotation of the spine. Several different approaches have been taken to assess and treat it. Although there have been many descriptions of the vertebral rotation, there have been few descriptions ${ }^{1-3}$ of the rotation of the spinal cord in scoliosis.

The purpose of this study was to analyse rotation of the spinal cord in patients with idiopathic scoliosis and to establish its clinical significance.

\section{Patients and Methods}

Of patients with scoliosis awaiting surgery who underwent a pre-operative, standing radiograph and computed tomographic myelography (CTM), 78 with a right thoracic idiopathic scoliosis were studied. Those in whom the conus medullaris was cephalad to the apical region (all King type I) were excluded. Following myelography with lumbar puncture using a 23-gauge spinal needle, CT scans (GE High Light Advantage, GE Medical Systems, Waukesha, Wisconsin) $140 \mathrm{kV}, 100 \mathrm{~mA}, 2$ seconds or Siemens Somatom Plus 4, 140kV, $171 \mathrm{~mA}, 1$ second (Siemans AG, Medical Solutions, Erlangen, Germany) were obtained. The purpose of myelography and CTM was to investigate accurately the neuraxis, the position of the dural sac and spinal cord, the rib cage proportion, and the vertebral anatomy.

At the occipitocervical, cervicothoracic, apical region, conus area, and S1 levels, the CT

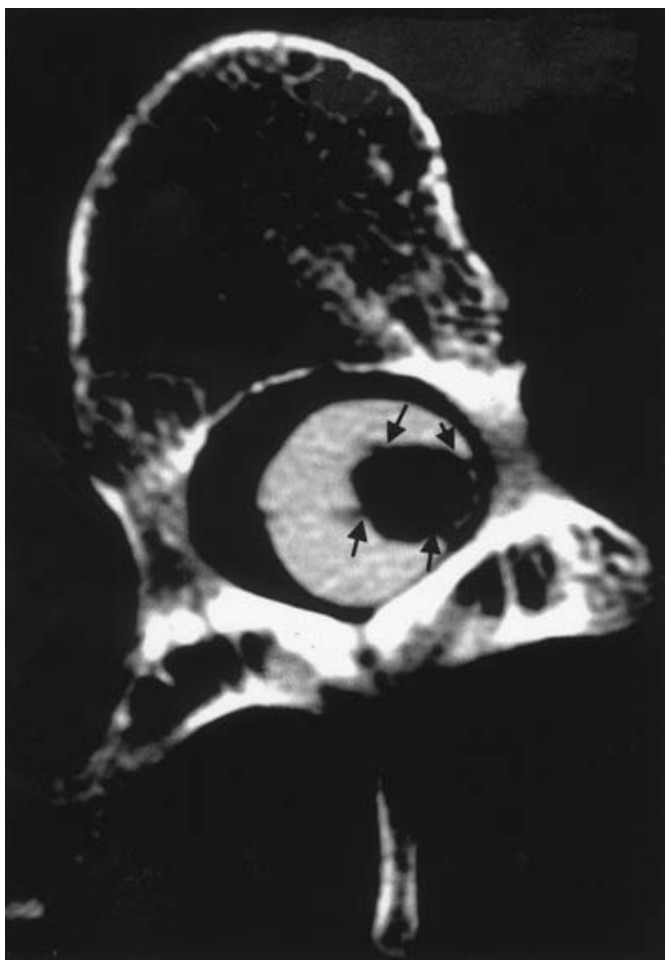

Fig. 1

Sample CT scan used to calculate the angle of rotation of the spinal cord. The arrows indicate the origins of four nerve roots on the surface of the spinal cord.

sections were $2 \mathrm{~mm}$ thick and $2 \mathrm{~mm}$ apart. We took special care to ensure that the CT gantry was aligned parallel to the axis of each pedicle. If the CT gantry slanted more than $5^{\circ}$ toward 


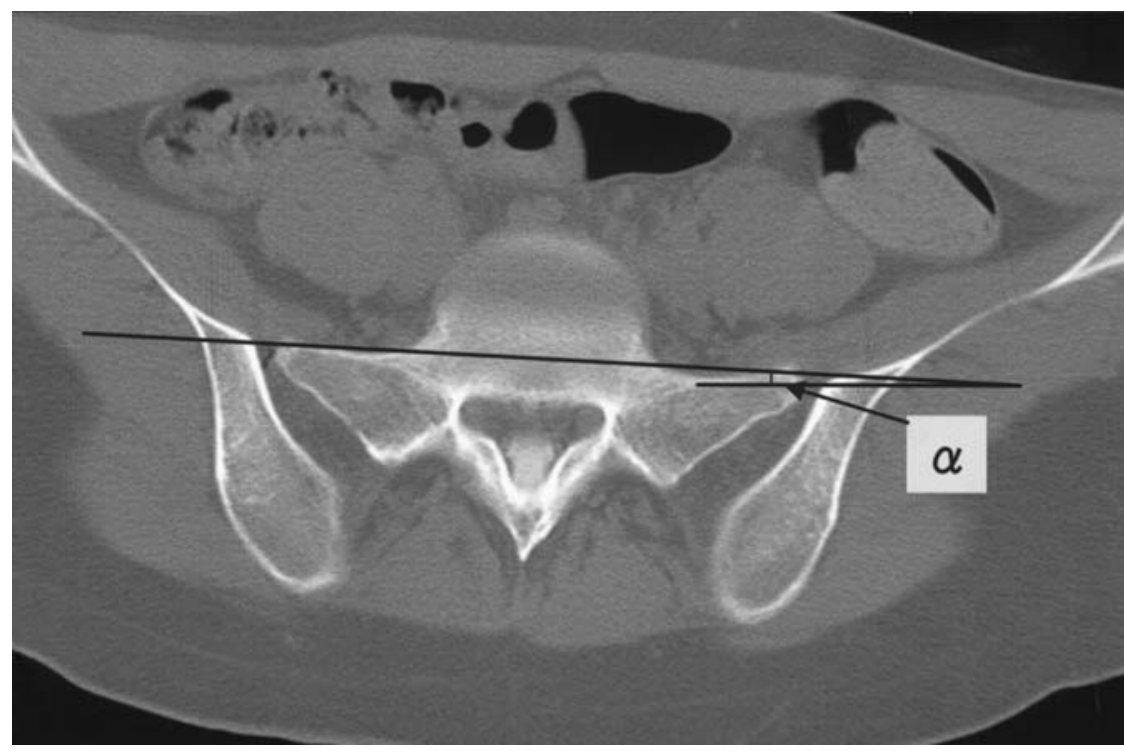

Fig. 2

CT scan showing the method of calculating the angle of rotation of the S1 vertebral body ( $\alpha$, the tilt of S1).

the axis of both pedicles, a three-dimensional reconstruction was performed. We were then able to obtain a new slice parallel to the axis of each pedicle.

Morphometric parameters were measured in the apical vertebral slice, including the angle of rotation of the spinal cord and vertebra. The method of measurement of the angle of rotation of the spinal cord is shown in Figure 1. Four points were located on the origins of each pair of nerve roots on the surface of the spinal cord. A line connecting the two anterior points was considered to be the ventral line and a line connecting the two posterior points was considered to be the dorsal line. The line bisecting these two lines was designated the inherent transverse axis of the spinal cord. The tilt of this axis was modified by that of $\mathrm{S} 1$ (Fig. 2) and was thereafter called the angle of rotation of the spinal cord (RAc). In Figure 3, the method of calculating the angle of rotation of the vertebral body (RAsag) is shown. ${ }^{4}$ RAsag was also modified by the tilt of $\mathrm{S} 1$, and was thereafter called RAsac.

In some cases, especially in severe scoliosis, we could not find the origins of the nerve roots on the concave side because the spinal cord was too deviated and the subarachnoid space was diminished. In this situation, we looked for the datum points in two other slices in which the origins of the nerve roots could be identified both cephalad and caudal at the same vertebral level. The mean value of the angles in these two slices was measured. A plain, standing radiograph was used to measure the Cobb angle and to assign the King curve type.

Statistical analysis was performed using Stat View statistical software version 5.0 (SAS Institute Inc, Cary, North
Carolina). Student's $t$-test and Pearson's correlation coefficient were used. The criterion for statistical significance was $\mathrm{p}<0.05$.

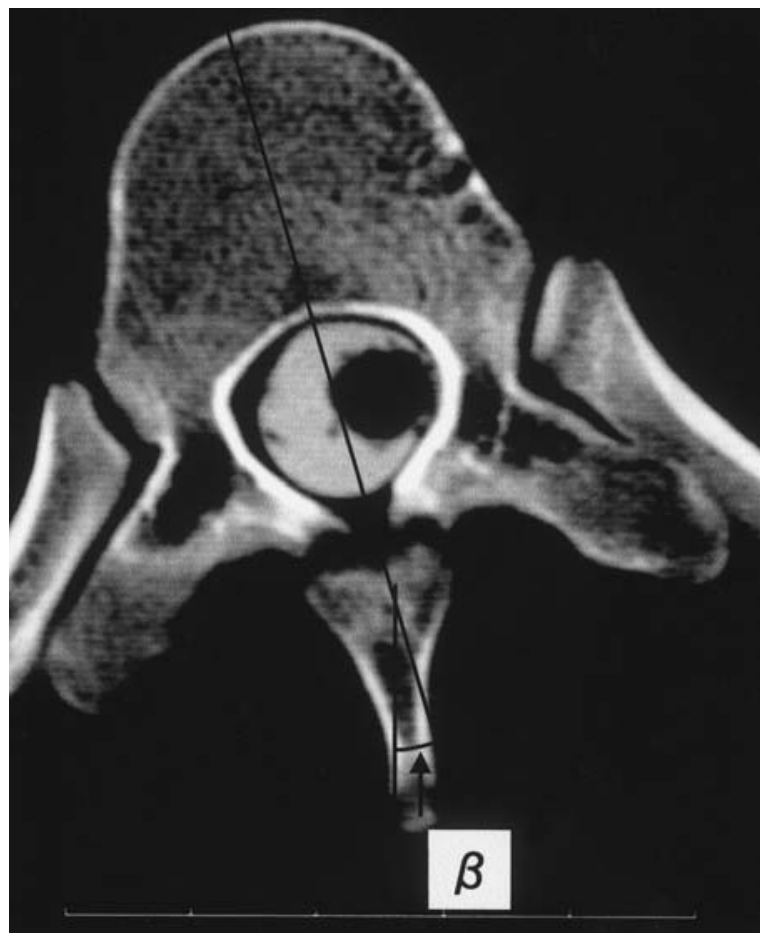

Fig. 3

CT scan showing the method of calculating the angle of rotation of the vertebral body ( $\beta$, RAsag) ${ }^{4}$ 


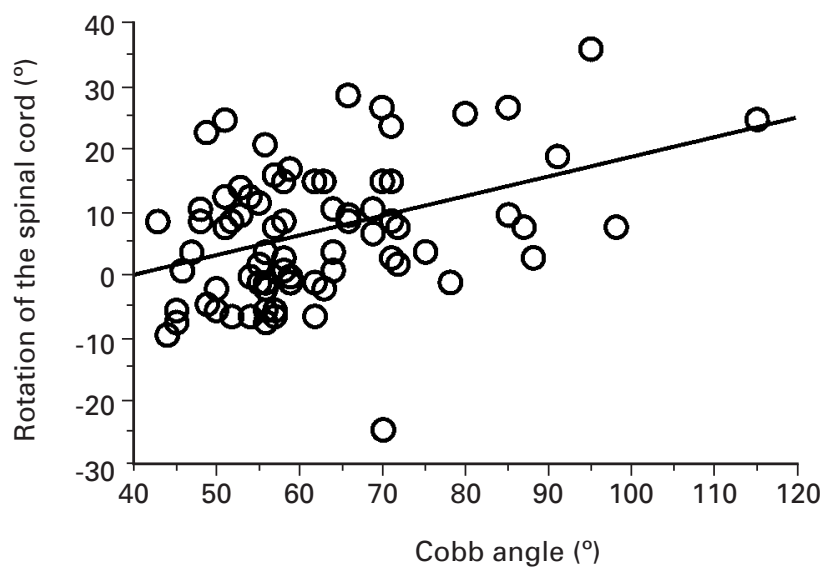

Fig. 4

Correlation between the rotation of the spinal cord (RAc) and the Cobb angle $(p<0.0005 ; r=0.395)$.

\section{Results}

Of the 78 patients, 72 were women and six were men. The mean age at which the patients underwent CTM was 15.8 years (10.6 to 25.9 ) and the mean Cobb angle was $62.3^{\circ}$ (43 to 115). Eighteen patients had King type II curves, 32 type III, 17 type IV, and 11 type V. RAc had a statistically significant relationship with both RAsac and the size of the primary curve (Figs 4 and 5). RAc was significantly smaller than RAsac. Age and gender were independent of the Cobb angle, RAc and RAsac.

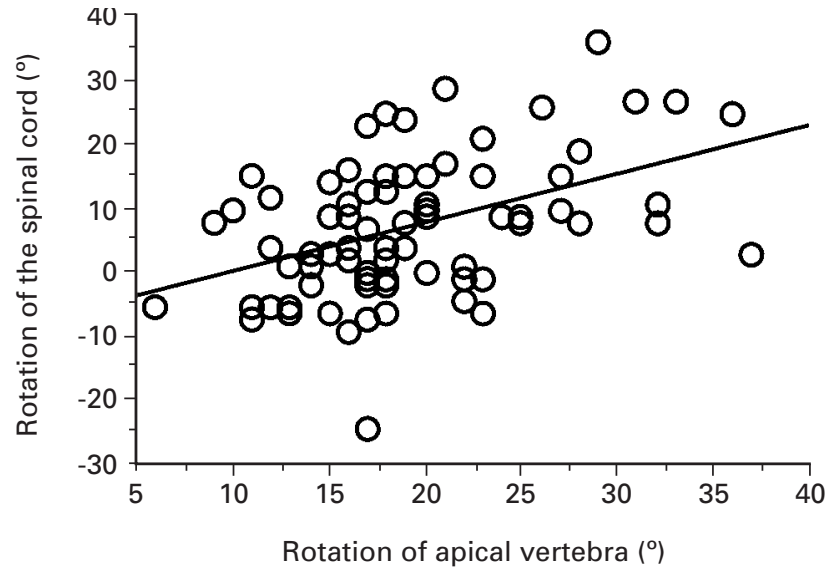

Fig. 5

Correlation between the rotation of the spinal cord (RAc) and that of the apical vertebra $(p<0.0001 ; r=0.443)$.

We have developed a system for classifying the relationship between the rotation of the spinal cord and that of the vertebral body in the apical region. These relationships can be divided into three distinct groups (Fig. 6). In the overrotation $(\mathrm{O})$ type, the spinal cord and the apical vertebral body are rotated in the same direction, and RAc is more than RAsac. In the under-rotation (U) type, the spinal cord and the apical vertebral body are rotated in the same direction, and RAc is less than RAsac. In the reverse rotation (R) type, the spinal cord and apical vertebral body are rotated

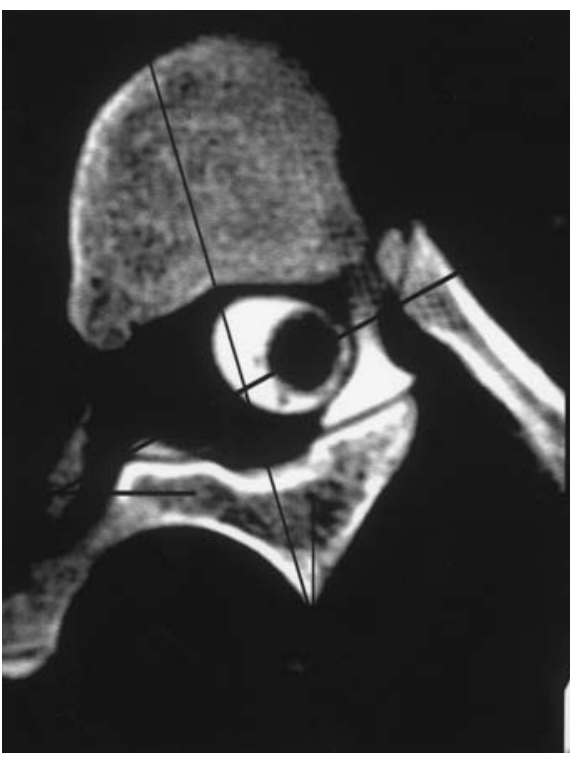

Fig. 6a

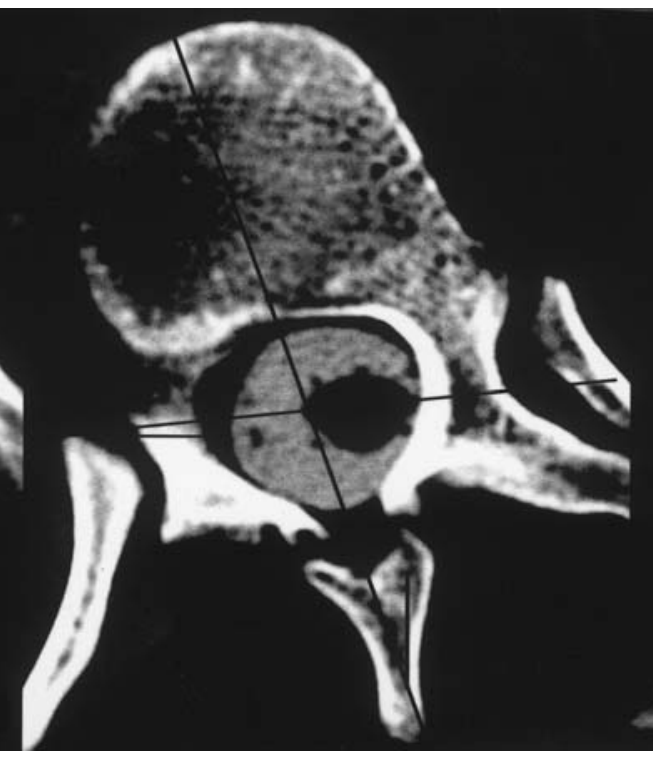

Fig. 6b

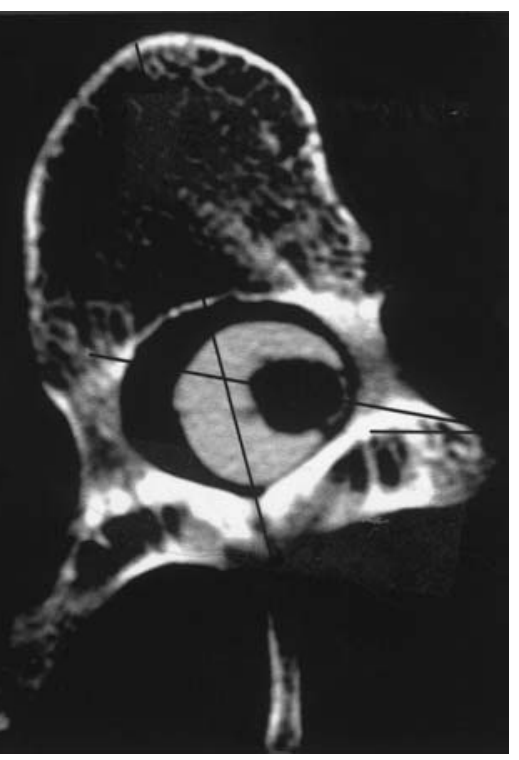

Fig. 6c

CT scan showing the rotational types of relationship between the spinal cord and the apical vertebra a, over-rotation (O) type; b, under-rotation (U) type; $c$, reverse rotation $(R)$ type. 
Table I. The relationship between King-Moe curve types (II to V) and rotational types $(\mathrm{O}, \mathrm{U}$ and $\mathrm{R})$

\begin{tabular}{lcccl}
\hline & Type II & Type III & Type IV & Type V \\
\hline O type & 2 & 2 & 2 & 0 \\
U type & 13 & 15 & 15 & 6 \\
R type & 3 & 15 & 0 & 5 \\
\hline
\end{tabular}

in opposite directions. There were six patients with the $\mathrm{O}$ type, 49 with the U type, and 23 with the R type (Table I).

\section{Discussion}

Myelography and CTM are invasive in terms of radiation. Because of the difficulty in assessing MR images of the apical segments ${ }^{5}$ and because of the artefacts created by the pulsation of cerebrospinal fluid and the distortion, we used myelography and CTM in order to analyse the neuraxis accurately.

Hilal and Keim, ${ }^{1}$ undertook selective spinal angiography in 33 patients with scoliosis. Based on the finding that the anterior spinal artery lay closer to the posterolateral artery near the concave side of the spine, they concluded that the spinal cord and vertebral bodies rotated in opposite directions. This conclusion has been cited by several authors, ${ }^{2,6}$ but has not been verified for a long time. In our series, the spinal cord and apical vertebral body were rotated in opposite directions (R type) in only one third of the patients. In the anteroposterior view of the $\mathrm{R}$ type, the anterior spinal artery is near the posterior spinal artery on the concave side (Fig. 7a). In the U type, especially when the spinal cord deviates so much as to be compressed by the concave pedicle, we also found that the anterior spinal artery was near to the posterior spinal artery on the concave side in the anteroposterior view (Fig. 7b). If only spinal angiography is performed, these two conditions can be indistinguishable. The discrepancy between our results and previous reports may have occurred because selective spinal angiography only reveals the vascular components and cannot show the total shadow of the spinal cord.

In normal anatomy, the spinal cord is anchored to the arachnoid by the arachnoid meshwork ${ }^{7}$ and to the dura mater by the denticulate ligaments. The dura mater extends to surround the emerging nerves and becomes incorporated with their outer sheaths. The nerves thus act as guy ropes which may inhibit free rotation of the cord and its coverings. ${ }^{2}$ If the vertebrae rotate in one direction, the resulting torsion causes the spinal cord to rotate in the same direction although not necessarily to the same degree (U type). In contrast, because the dura mater is a tough, unyielding structure, the cord may rotate in the opposite direction to that of the vertebrae in the apical region because of the torsional strain which occurs in the cephalad compensatory curve ( $\mathrm{R}$ type). The discrepancy between these two types may be explained by differences in the tightness of the dura

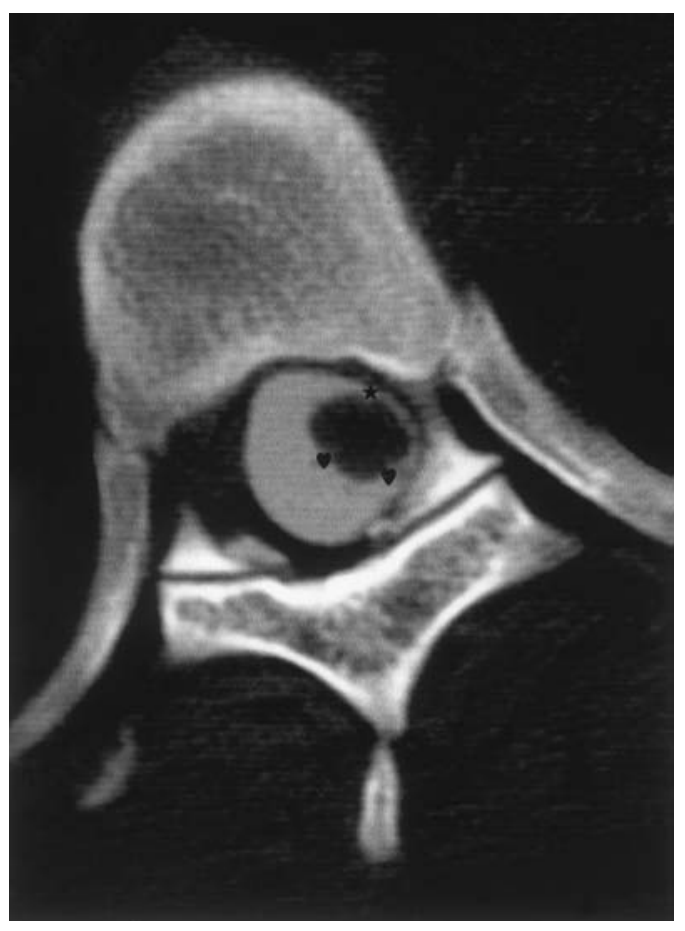

Fig. 7a

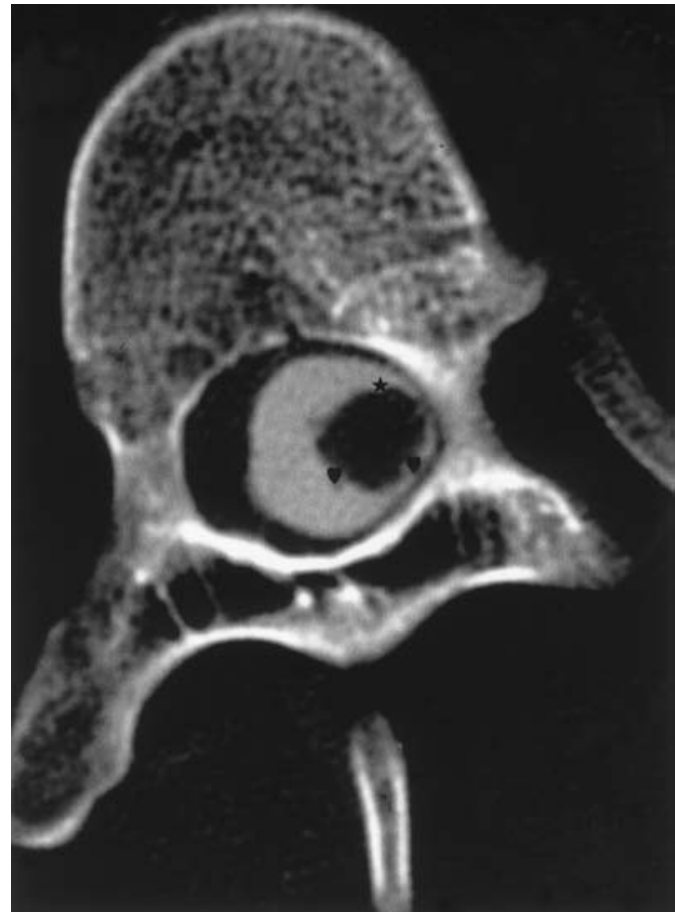

Fig. 7b

CT scans showing the positions of the anterior ( $\star$ ) and posterior ( $v$ ) spinal arteries a) in the $R$ type and b) in the $U$ type with cord compression. 
mater, or the spinal cord itself, between individuals. This hypothesis raises a question about why the spinal cord rotates more than the apical vertebra in the $\mathrm{O}$ type. There may be unknown causes for this kind of rotation.

The question of whether torsion of the spinal cord may cause a neurological deficit has been previously discussed. Steindler in his discussion in McKenzie and Dewar ${ }^{8}$ argued that torsion of the spinal cord was a more important cause of ischaemia than compression. Lloyd-Roberts et al, ${ }^{2}$ suggested that failure of the spinal cord to rotate with the vertebral column might cause intercostal nerve impairment. This might, in turn, be a factor in the progression of scoliosis. These observations suggest that rotation of the spinal cord might interrupt the blood supply or neural activity. Torsion may exacerbate other risk factors, such as kyphosis, distraction of the cord, and vascular damage.

As mechanical deformation can affect the function of the spinal cord, careful attention must be paid to this during the operative treatment of scoliosis, especially in the rod rotation manoeuvre. Machida et al, ${ }^{9}$ reported that the spinal cord was more vulnerable to a combined distraction and derotation manoeuvre than to either alone. Ghanem et al, ${ }^{10}$ analysed the effects on the vertebrae which were produced solely by rotation of the rod intra-operatively. They showed an increased apical vertebral rotation after rotation of the first rod in surgery for a thoracic scoliosis. Our results suggest that the deterioration of apical vertebral rotation may cause increased spinal cord rotation. Particularly for $\mathrm{O}$ type, it is possible that the stresses which are caused by manoeuvres of an over-rotated spinal cord can produce ischaemia or interruption of neural activity.

A $1.47 \%$ incidence of neurological complications when using Cotrel-Dubousset instrumentation (Medtronic Inc, Minneapolis, Minnesota) was reported in the Morbidity and Mortality of the Scoliosis Research Society (1988). ${ }^{9}$ Severe scoliosis carries an increased risk of neurological injury. ${ }^{11}$ Although the relationship between rotation of the spinal cord and neurological complications remains unclear, careful attention must be paid to this during the operative treatment of scoliosis. Our study has demonstrated that there are three types of rotational relationship between the spinal cord and vertebra at the apex of an idiopathic scoliosis. Further studies are clearly necessary in order to establish the pathophysiological significance of this.

We would like to thank Y. Fukuchi for his assistance with radiographic analysis and Dr N. Suzuki, Saiseikai Chuo Hospital, Japan, for his suggestions and advice.

No benefits in any form have been received or will be received from a commercial party related directly or indirectly to the subject of this article.

\section{References}

1. Hilal SK, Keim HA. Selective spinal angiography in adolescent scoliosis. Neuroradiology 1972;102:349-59.

2. Lloyd-Roberts GC, Pincott JR, McMeniman P, Bayley IJL, Kendall B. Progression in idiopathic scoliosis: a preliminary report of a possible mechanism. J Bone Joint Surg [Br] 1978;60-B:451-60.

3. Tuli SK, Hurlbert RJ, Mikulis D, Fleming JF. Ninety-degree rotation of the thoracic spinal thecal sac: case report. J Neurosurg 1998;89:133-8.

4. Aaro S, Dahlborn M. Estimation of vertebral rotation and the spinal rib cage deformity in scoliosis by computer tomography. Spine 1981;6:460-7.

5. O'Brien MF, Lenke LG, Bridwell KH, Blanke K, Baldus C. Pre-operative spinal canal investigation in adolescent idiopathic scoliosis curves $\geq 70^{\circ}$. Spine 1994;19: 1606-10.

6. Porter RW. The pathogenesis of idiopathic scoliosis: uncoupled neuro-osseous growth? Eur Spine J 2001;10:473-81.

7. Roth M. Caudal end of the spinal cord: I. normal pneumographic features. Acta Radiol Diagn 1965;3:177-88.

8. McKenzie KG, Dewar FP. Scoliosis with paraplegia. J Bone Joint Surg [Br] 1949; 31-B:162-74.

9. Machida M, Weinstein SI, Imamura Y, et al. Compound muscle action potentials and spinal evoked potentials in experimental spine manoeuvre. Spine 1989;14: 687-91.

10. Ghanem IB, Hagnere F, Dubousset JF, et al. Intra-operative optoelectronic analysis of three-dimensional vertebral displacement after Cotrel-Dubousset rod rotation. Spine 1997;22:1913-21.

11. MacEwen GD, Bunnell WP, Srimam K. Acute neurological complication in the treatment of scoliosis: a report of the Scoliosis Research Society. J Bone Joint Surg [Am] 1975:57-A:404-8. 